\title{
Spatial and Temporal Factors Determining Afferent Fiber Responses to a Grating Moving Sinusoidally Over the Monkey's Fingerpad
}

\author{
A. W. Goodwin, K. T. John, K. Sathian, ${ }^{\mathrm{a}}$ and I. Darian-Smith \\ Department of Anatomy, University of Melbourne, Parkville, Victoria 3052, Australia
}

\begin{abstract}
Gratings of alternating grooves and ridges were moved sinusoidally across the fingerpads of anesthetized monkeys, while responses were recorded from individual slowly adapting afferents (SAs), rapidly adapting afferents (RAs), and Pacinian afferents (PCs) in the median nerve. The stimulus comprised 2 spatial variables, namely, groove width $(G)$ and ridge width $(W)$, and 2 temporal variables, namely, the peak speed of movement $(S)$ and the peak temporal frequency $(F)$ at which successive spatial cycles of the grating pass over a point in the receptive field. The responses of all 3 fiber types were determined by only 1 spatial variable, G, and only 1 temporal variable, $F$. Changes in $W$ or $S$ affected responses only if there was a concomitant change in either $G$ or $F$. Responses were phase-locked to the occurrence of successive spatial cycles of the grating, and we have used the number of impulses elicited by a single spatial cycle as the fundamental measure of response. An equation of the form $I=c G a \exp (\quad b V \boldsymbol{F})$ describes the responses of all 3 fiber types. For SAs, the effect of groove width was greater $(a=2.64)$ than for RAs and PCs $(a=0.924$ and 1.05 , respectively). The reduction in response with frequency was most marked for SAs $(b=0.262)$, and greater for PCs $(b=$ $0.167)$ than for RAs $(b=0.130)$. From the equation, the instantaneous response during the entire sinusoidal cycle was reconstructed as well as a second measure, the mean cyclic response. These 2 measures behaved differently with changes in the stimulus parameters. The temporal properties of the fibers, as revealed by gratings, may appear to be in conflict with those established by vibratory threshold studies; in fact, they are compatible with suprathreshold responses to vibrating probes.
\end{abstract}

When cutaneous mechanoreceptors are activated, for example by scanning the fingertips over a surface, the stimulus is characterized by both temporal and spatial features. Many studies have used single probes, indenting or vibrating the skin, to define the temporal properties of the primary afferent fibers. These experiments delineated the responses of the different types of

\footnotetext{
Received Mar. 14, 1988; revised July 28, 1988; accepted Aug. 25, 1988.

This work was supported by a grant from the National Health and Medical Research Council of Australia. We thank Neil Ratcliffe, Colin Clarke, and Alf Medoro for technical assistance.

Correspondence should be addressed to Dr. A. W. Goodwin, Department of Anatomy, University of Melbourne, Grattan Street, Parkville, Victoria 3052 , Australia.

Present address: Department of Anatomy and Neurobiology, Washington University School of Medicine, St. Louis, MO 63110.

Copyright (C) 1989 Society for Neuroscience $0270-6474 / 89 / 041280-14 \$ 02.00 / 0$
}

mechanoreceptors to time-varying stimuli, defined either directly by their temporal components (Knibestöl, 1973; Pubols and Pubols, 1976) or, more commonly, by their frequency components (Talbot et al., 1968; Freeman and Johnson, 1982; Johansson et al., 1982a). The spatial properties of the afferents were initially examined by altering the position of indenting or vibrating probes within the receptive field (Johnson, 1974; Johansson, 1978). More complex spatial arrangements such as 2 vibrating probes (Goodwin et al., 1981) and indenting gratings (Phillips and Johnson, 1981a) have also been used. In most natural stimuli, temporal and spatial attributes are linked. Experimental stimuli of this type have included dot patterns (Lamb, 1983) and curved surfaces (LaMotte and Srinivasan, 1987a, b) scanned across the fingerpads. At the cortical level, neurons have also been examined using toothed wheels rolled across the skin (Warren et al., 1986).

The stimulus that we have found particularly useful is a grating of alternating grooves and ridges scanned sinusoidally across the fingerpad. This motion mimics the movement used by human subjects exploring textured surfaces. A major advantage of such a stimulus is that both the temporal and the spatial components of the moving grating can be easily specified and controlled. (The stimulus, as used by us, refers to the moving grating; resultant changes in skin parameters, such as indentation and the various stress components, are pursued in the Discussion.) However, so far we have only empirical descriptions of the afferent responses and no complete analytical model, so that it is not possible to synthesize information from our various studies or to relate them to experiments that used different stimuli (Darian-Smith and Oke, 1980; Goodwin and Morley, 1987a, b; Morley and Goodwin, 1987; Sathian et al., 1989). Three examples of these deficiencies follow. First, when the spatial period of a grating changes, is the change in response documented by Goodwin and Morley (1987a) simply a reflection of the change in groove width as suggested by Sathian et al. (1989)? Second, for vibratory stimuli, afferent responses have minimum threshold amplitudes at a particular frequency (Talbot et al., 1968), but such tuning is not apparent in the responses to gratings. Third, changes in stimulus parameters change the profile of the response during the sinusoidal cycle. Thus, the various response measures used, such as the mean cyclic response and the magnitude of the phase-locked response, are affected differently by changes in stimulus parameters; it is currently not possible to reconcile these differences. In this paper we develop a mathematical description of the responses of the different afferent types to gratings moving sinusoidally. With this precise description of the underlying spatial and temporal 
factors, it is possible to synthesize previous studies and to eliminate many of the apparent contradictions that currently exist.

\section{Materials and Methods}

Gratings of alternating grooves and ridges were moved sinusoidally across the receptive fields of cutaneous mechanoreceptive afferents in anesthetized monkeys. Responses were recorded from 13 slowly adapting afferents (SAs), 17 rapidly adapting afferents (RAs), and 7 Pacinian afferents (PCs), all with receptive fields central located on a fingerpad. These are the same afferents that were studied in the previous paper (Sathian et al., 1989), where the recording methods are described in detail.

The sinusoidal movement, which had a peak-to-peak amplitude of $80 \mathrm{~mm}$, resulted in a speed profile that was a rectified sinusoid and whose peak value depended on the temporal period of the cycle. At any instant of time during the motion, successive spatial cycles of the grating passed over the receptive field at an instantaneous rate that we call the temporal frequency of the grating spatial cycles. This frequency is a complicated function of the spatial period of the grating and the temporal period of the sinusoid (Morley and Goodwin, 1987). During the central $42^{\circ}$ of each half of the movement cycle, the speed was constant to within $6.6 \%$ of its peak value $(S)$, and thus the temporal frequency of the grating spatial cycles was constant to within $6.6 \%$ of its peak value $(F)$. These 2 parameters and the spatial period of the grating $(P)$ are related by the expression $S=P F$. A mathematical description of the stimulus is given in the Appendix.

Thirty-eight gratings were used. There were 2 blocks in which ridge width was nominally constant $(0.3$ and $1.5 \mathrm{~mm})$ and 6 blocks in which groove width was nominally constant $(0.25,0.5,1,1.25,1.5$, and 2 $\mathrm{mm})$. The actual values of the grating parameters are given in Table 1 of the previous paper. In the first series of experiments, the period of the sinusoid was held constant at $3.14 \mathrm{sec}$, resulting in a peak speed of movement of $80 \mathrm{~mm} / \mathrm{sec}$. Responses of RAs were recorded for all 8 blocks of gratings. For PCs, the block with a groove width of $0.25 \mathrm{~mm}$ was omitted, and for SAs, the blocks with groove widths of 0.25 and $0.5 \mathrm{~mm}$ were omitted, as responses to these gratings could not be distinguished from responses to a smooth surface (Sathian et al., 1989). Some data were also collected using an additional 13 gratings in which the spatial period was nominally constant at $1.5,2$, or $3 \mathrm{~mm}$. In the second series of experiments, the temporal period of the sinusoid, and hence the peak speed of movement, was varied in order to set the peak temporal frequency of the grating spatial cycles $(F)$ at 20,40 , or $80 \mathrm{~Hz}$.

\section{Results}

During the portion of the movement cycle when speed is approximately constant (central $42^{\circ}$ ), the responses of the afferents are phase-locked to the passage of successive spatial cycles of the grating over the receptive field (Morley and Goodwin, 1987). Thus, a measure of the responsiveness of an afferent is given by the number of impulses elicited by the passage of a single spatial cycle of the grating. There are 2 regions of constant speed; the first when the grating moves over the receptive field in one direction, and the second when the grating moves in the opposite direction, and the responses of most afferents are different for the 2 directions (Goodwin and Morley, 1987b). We calculated the number of impulses per grating spatial cycle averaged over both regions and over many trials. As will be seen, this response measure provides the brain with additional information that is different from that provided by the mean cyclic response used previously. More importantly, the number of impulses in each burst can be treated as a fundamental response measure, and more complex features such as the discharge profile during a cycle, or the mean cyclic response, can be derived from it.

\section{Responses when peak speed of movement remained constant}

Gratings were presented in blocks within which either the ridge width or the groove width remained constant. Responsiveness varied greatly among the afferents. As an illustration, consider the SAs responding to gratings with a ridge width of $0.3 \mathrm{~mm}$. All SAs had responses that increased monotonically as the groove width of the grating increased, but there was a large spread of response magnitudes. To eliminate the effect of different sensitivities, responses were divided by a normalizing factor before pooling. The normalizing factor was the average response to the 14 gratings in the blocks, groove $=1.25 \mathrm{~mm}$, groove $=2 \mathrm{~mm}$, and ridge $-0.3 \mathrm{~mm}$. The mean normalized responses are shown in Figure $1 A$ (left).

Figure 1 shows the mean normalized responses for both the constant ridge width blocks (left) and the constant groove width blocks (right) for all 3 classes of mechanoreceptive afferents. For the RAs and PCs the normalizing factor was the average response to the 11 gratings in the blocks, groove $=1 \mathrm{~mm}$ and groove $=2 \mathrm{~mm}$. The essential features of the responses are as follows. For all curves the SEs are small. Thus, the underlying relationship between responses and the grating parameters was the same for all afferents within each class, despite large variations in the sensitivity of individual afferents. When ridge width remained constant (left column), response increased monotonically as a function of groove width for all 3 fiber types. Similarly, when groove width remained constant (right column), response increased monotonically as a function of ridge width. Thus, the number of impulses per grating spatial cyclc bchaved differently from the mean cyclic discharge rate, which is the measure used previously (Goodwin and Morley, 1987a; Sathian et al., 1989). This is readily seen by comparing Figure 1 with Figure 3 of the accompanying paper. Both measures increased with increasing groove width (left). However, when ridge width increased (right), the number of impulses per grating spatial cycle increased but the mean cyclic response did not.

\section{Responses when peak temporal frequency of the grating spatial cycles remained constant}

The data in Figure 1 alone are insufficient to define quantitatively the relationship between responses and the grating parameters. This is because a temporal parameter covaried with the spatial parameters of the grating. When the peak speed $(S)$ remained constant, the peak temporal frequency of the grating spatial cycles $(F)$ covaried with changes in the spatial period of the grating $(P)$ according to the relationship $S=P F$. Thus, changes in groove width or ridge width in Figure 1 were accompanied by changes in $F$, and it is not possible to isolate the contributions of the spatial and temporal factors. Therefore, a second set of experiments was performed. The responses of the afferents were measured as a function of groove width and ridge width while $F$ remained constant. Obviously the peak speed was different for different gratings. For each class of fibers, data were collected for 1 block of constant ridge width $(0.3 \mathrm{~mm})$ and 2 blocks of constant groove width (1.25 and $2 \mathrm{~mm}$ for the SAs, and 1 and $2 \mathrm{~mm}$ for the RAs and P(s). Two values of constant $F$ were used, 20 and $40 \mathrm{~Hz}$ for the SAs, and 40 and $80 \mathrm{~Hz}$ for the RAs and PCs.

Figure 2 shows mean normalized responses for the 3 fiber types as a function of groove width (left) and of ridge width (right). The most striking feature of this figure is that when $F$ remained constant, ridge width (right) had essentially no effect on response. Moreover, the curve for the higher value of $F$ is always well below the curve for the lower value of $F$, so that response decreased as $F$ increased. The same result held when groove width was constant at $2 \mathrm{~mm}$ (not illustrated). Hence it 


\section{A. Slowly adapting afferents}
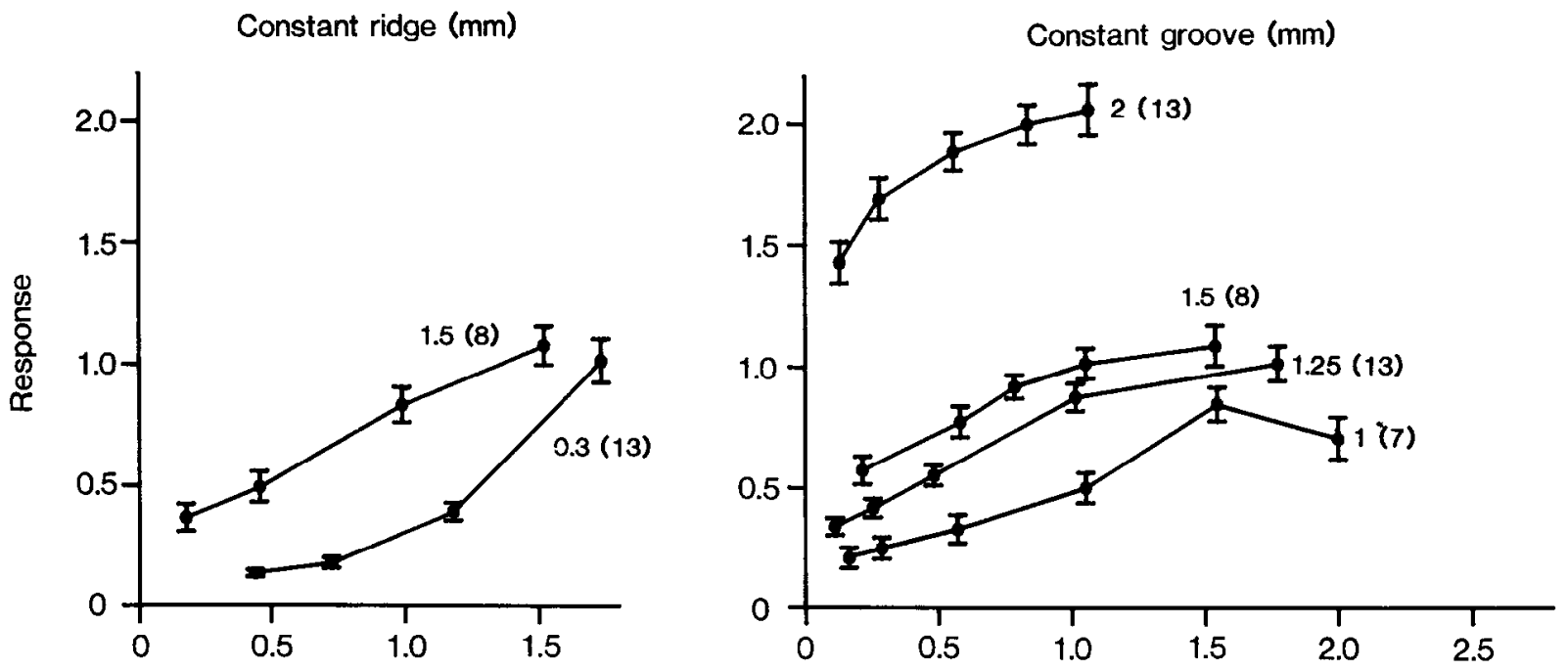

B. Rapidly adapting afferents
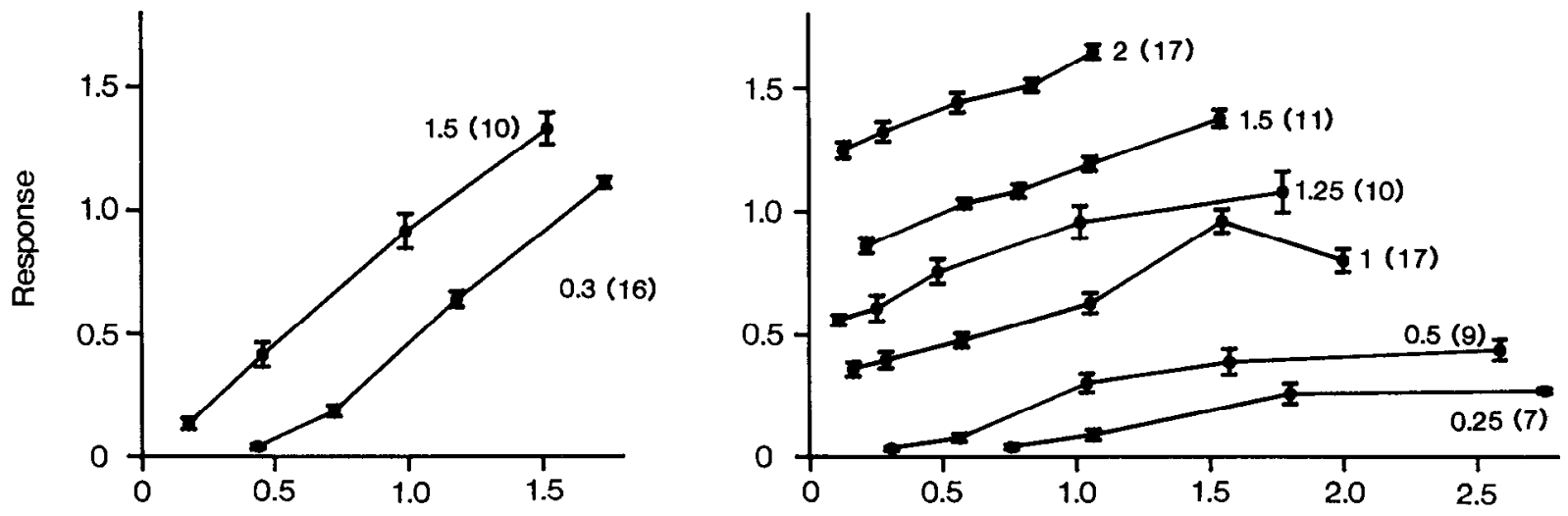

\section{Pacinian afferents}
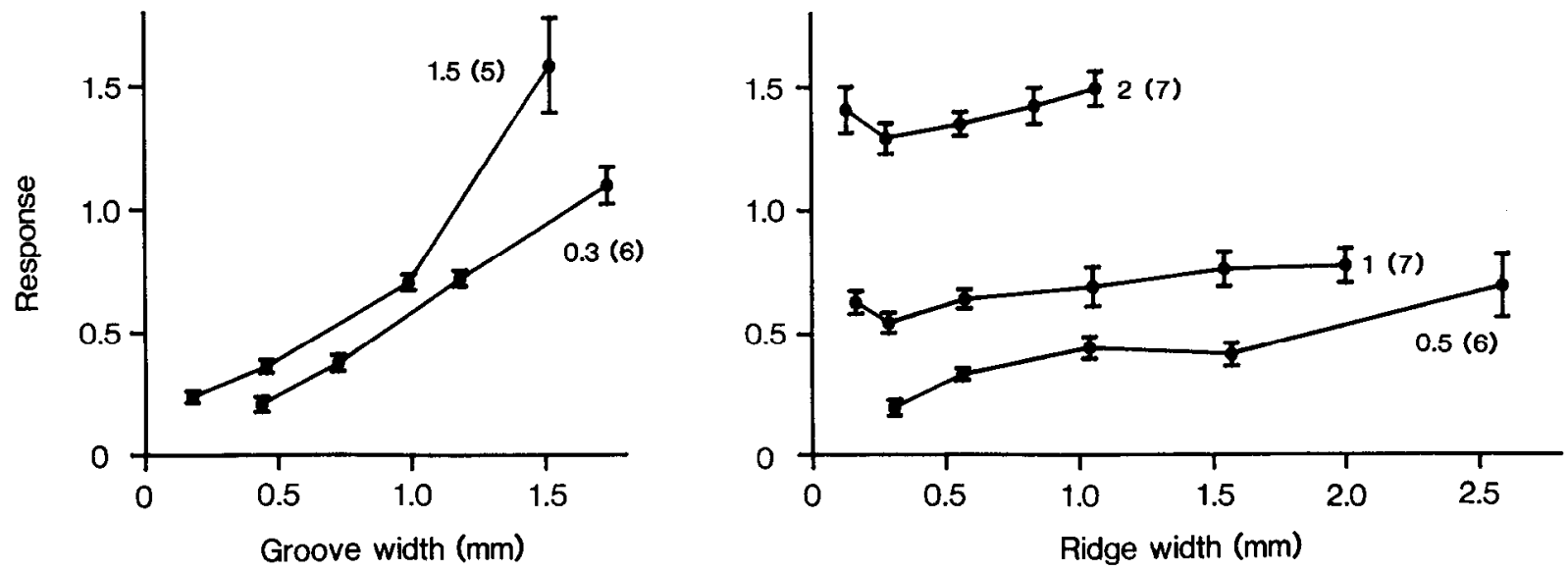

Figure 1. Responses of the 3 fiber types when peak speed remained constant at $80 \mathrm{~mm} / \mathrm{sec}$. In addition, either ridge width remained constant (left column) or groove width remained constant (right column) at the value (in $\mathrm{mm}$ ) indicated next to each curve. Response is the mean normalized number of impulses per grating spatial cycle, averaged over the 2 regions of movement when speed is nearly constant. The number of afferents in each block is indicated by the number in parentheses, and error bars indicate \pm 1 SEM. 


\section{A. Slowly adapting afferents}

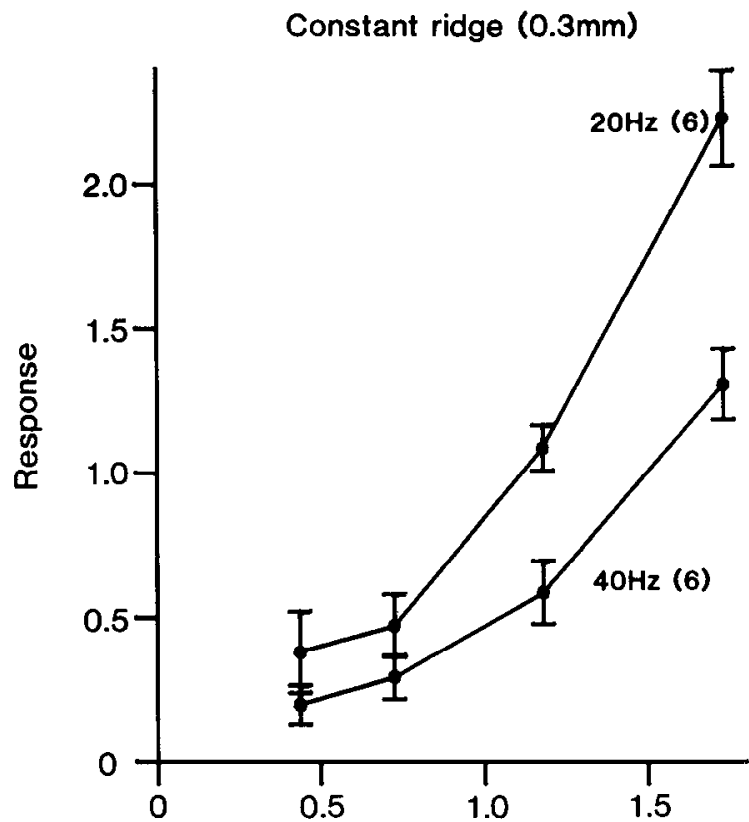

B. Rapidly adapting afferents

Constant ridge $(0.3 \mathrm{~mm})$

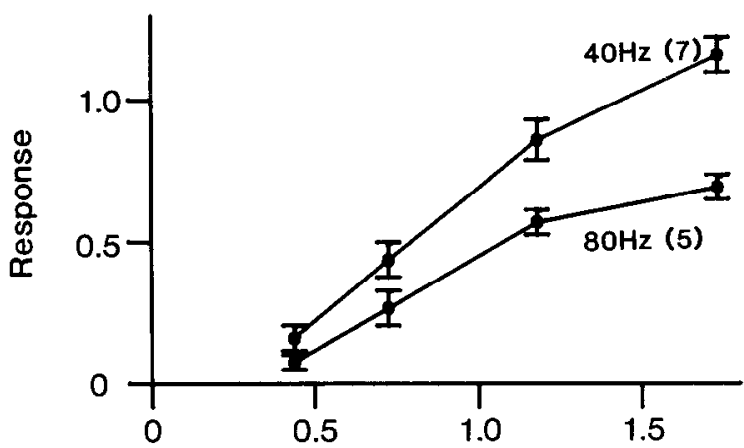

C. Pacinian afferents

Constant ridge $(0.3 \mathrm{~mm})$

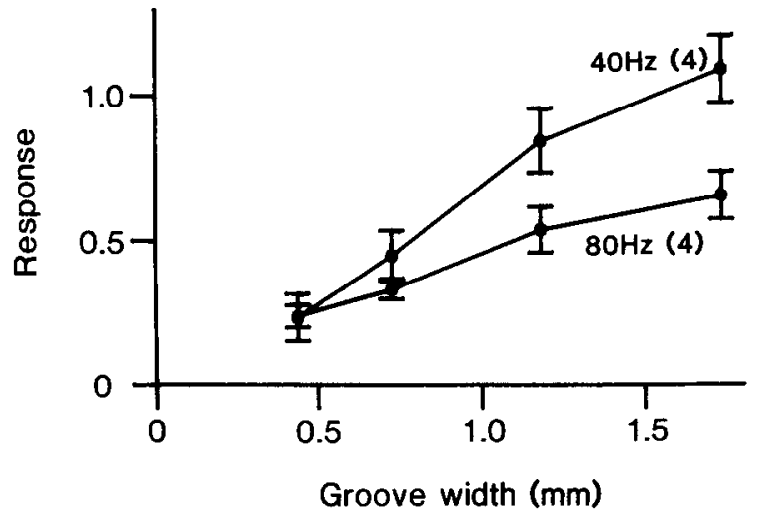

Constant groove $(1.25 \mathrm{~mm})$

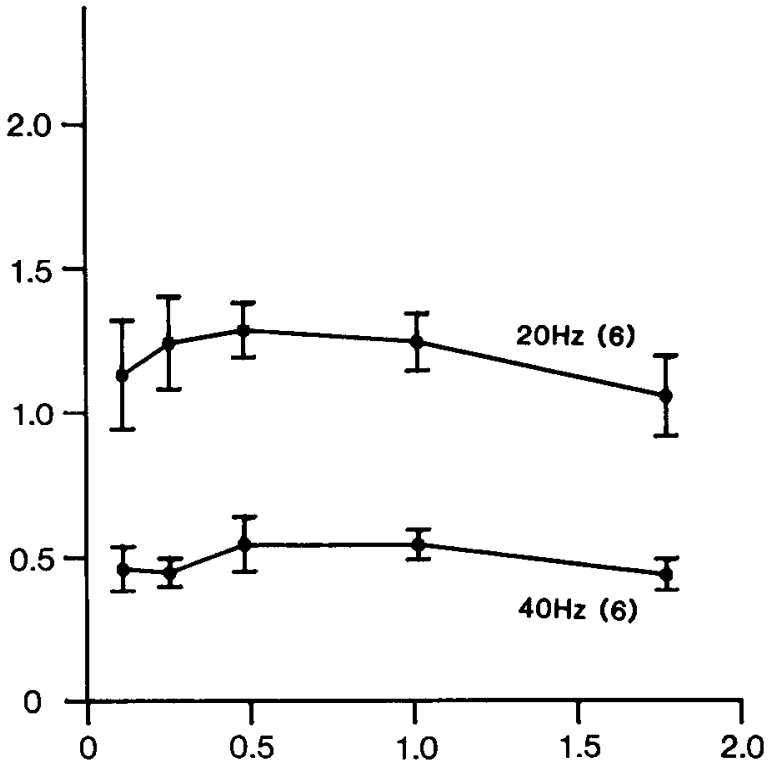

Constant groove $(1 \mathrm{~mm})$

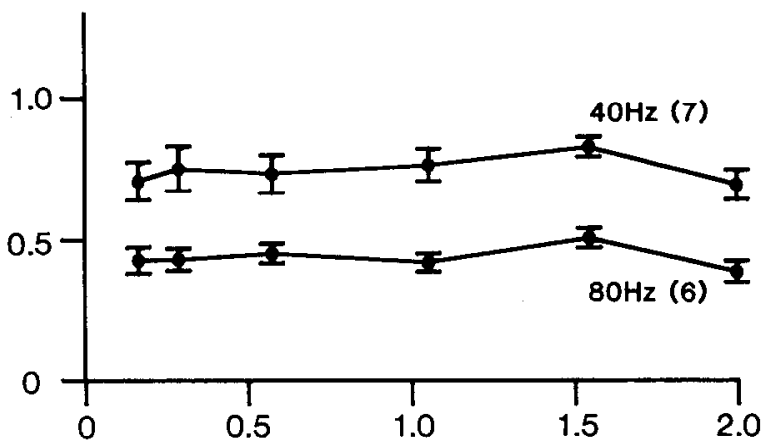


Table 1. Regression of $I_{\text {inc }}^{\prime}=c G^{a} \exp (-b \vee F)$ for the 3 fiber types

\begin{tabular}{lccc}
\multicolumn{4}{l}{ Fiber type } \\
\cline { 2 - 4 } & SA & RA & PC \\
\hline$a$ & 2.64 & 0.924 & 1.054 \\
$b$ & 0.262 & 0.130 & 0.167 \\
$c$ & 1.25 & 1.58 & 1.61 \\
$n$ & 583 & 785 & 429 \\
$f r$ & 0.824 & 0.833 & 0.791
\end{tabular}

The optimal values of the constants $a-c$ were obtained by direct nonlinear regression. For each regression, $n$ gives the number of data points, and $f r$ gives the fraction of the variance in response accounted for by the equation.

follows that the increase in response seen in Figure 1 (right) was not due to the increase in ridge width per se, but rather to the concomitant decrease in $F$. In contrast, an increase in groove width resulted in an increase in response, both when the peak speed remained constant (Fig. 1, left) and when the peak temporal frequency of the grating spatial cycles remained constant (Fig. 2, left). However, there is some difference in the shapes of these 2 sets of curves, implying a difference in the temporal factors for the 2 conditions.

\section{Mathematical description of response}

Inspection of Figures 1 and 2 immediately raises 3 questions. First, what are the natures of the functions relating responses to the various parameters? Second, how do these functions compare for the 3 fiber types? Third, how are the temporal factors in the blocks at constant speed related to those in the blocks at constant $F$ ? Such questions can only be answered by a mathematical derivation of the functional relationship between the various parameters.

There are 2 spatial variables, namely, the groove width $(G)$ and the ridge width $(W)$; and 2 temporal variables, namely, the peak speed $(S)$ and the peak temporal frequency of the grating spatial cycles $(F)$. In our nomenclature, $S$ is a temporal variable since it is independent of $G$ and $W$, the spatial variables of the grating. However, these 4 variables must also satisfy the equation $S=(G+W) F$. Thus, there are only 3 independent variables; the response can be expressed as a function of any 3 of these 4 variables. The simplest expression results from considering response as a function of $G, W$, and $F$. Because responses did not vary (as a function of $W$ ) when both $G$ and $F$ remained constant (Fig. 2, right), it follows that response can be expressed as a function of $G$ and $F$ only. The choice of the actual functions is dictated by the following considerations.

Fibers in all 3 classes responded to a smooth surface. This indicates that extraneous factors, such as microtexture in the surfaces and vibrations in the stimulator, contributed to responses (see Discussion). For RAs this response was small compared with the responses to the gratings, but for SAs and PCs the response to the smooth surface was comparable to the responses to the finer gratings. This cannot be shown in Figures 1 or 2 as spatial cycles are undefined for a smooth surface, but it is clearly evident when mean cyclic response is used as the measure of activity [Fig. 3 of the previous paper (Sathian et al., 1989)]. Thus, responses are considered to have 2 components. The first, the background response, is equivalent to that produced by a smooth surface. The second component, the incremental response, is the additional response produced by the grooves and ridges of the grating.
Figure 2 (left) suggests that the effect of groove width on the incremental response can be accounted for by a power function of the form $G^{u}$, with different exponents $(a)$ for the different fiber types. As $G$ tends to 0 , the response tends to that produced by a smooth surface, that is, the background response. It is clear from Figure 2 (right) that an increase in $F$ results in a decrease in response; and replotting Figure 1 (right), with $F=S /(G+$ $W)$ as the abscissa, suggests that the effect of $F$ can be accounted for by an exponential function of the form $\exp (-b \vee F)$. As $F$ tends to infinity, the number of impulses per grating spatial cycle tends to 0 , and as $F$ tends to 0 , the number increases to a maximum but finitc valuc. Appropriatc bchavior of this function for the limiting values of $F$ is essential for the reconstruction of instantaneous responses throughout the cycle (see next section).

Based on the above considerations, we tested the, adequacy with which the incremental component of the number of impulses per grating spatial cycle could be described by a function given by

$$
I_{\text {inc }}^{\prime}=c G^{a} \exp (-b \vee F) .
$$

A rigorous mathematical derivation and a definition of all the terms used is contained in the Appendix. Direct nonlinear regression (Press et al., 1986) was used to obtain the optimum values of the constants $a, b$, and $c$, and to assess the validity of the model. For each fiber, all combinations of $G, W, F$, and $S$ that we used were included, resulting in 583 data points for regression of the SAs, 785 for the RAs, and 429 for the PCs. Table 1 gives the values of the constants for the 3 fiber types. A measure of the validity of the model is provided by comparing the variance in $I^{\prime}{ }_{\text {inc }}$ accounted for by the equation with the total variance in $I_{\text {inc }}^{\prime}$ (Burr, 1974). As seen in Table 1, this is a high fraction $(f r)$, verifying that the equation provides a good description of our data.

Superimposing responses predicted by the model on the experimental responses shows the extent to which the 2 correspond or deviate and allows individual assessment of the suitability of the power term $G^{a}$ and the exponential term, $\exp (-b \vee F)$. To avoid loss of clarity caused by crowding, the data in Figures 1 and 2 have been replotted in Figures 3 and 4, respectively, using open circles and broken lines (with SEs omitted). Corresponding responses predicted by the model are shown by solid lines. In these and all subsequent reconstructions, values for the model are computed using the actual grating parameters corresponding to the experimental data points, rather than using the nominal values (see Table 1 of previous paper). Because the actual values deviate slightly from the nominal values, the reconstructions are not perfectly smooth [e.g., in Fig. $3 A$ (right), the second point on the line, groove $=1.5 \mathrm{~mm}$, had an actual groove width of $1.42 \mathrm{~mm}$ ]. As seen in Figures 3 and 4, the major trends in the data are accounted for by the model for all 3 afferent types under all stimulus conditions. There are, of course, minor differences; for example, in Figure $4 A$ (right), the model predicts a slight increase in response with increasing ridge width (because of the background component), whereas the data suggest no change. In assessing these differences the uncertainties in the data, indicated by the standard errors, must be taken into account. In Figure 3 (right), $G$ remains constant and $F$ varies; the correspondence between the model and the data demonstrates the validity of the function $\exp (-b \vee F)$. In Figure 4 (left), $F$ remains constant and $G$ varies; the correspondence demonstrates the validity of the $G^{a}$ term. 


\section{A. Slowly adapting afferents}

Constant ridge $(\mathrm{mm})$

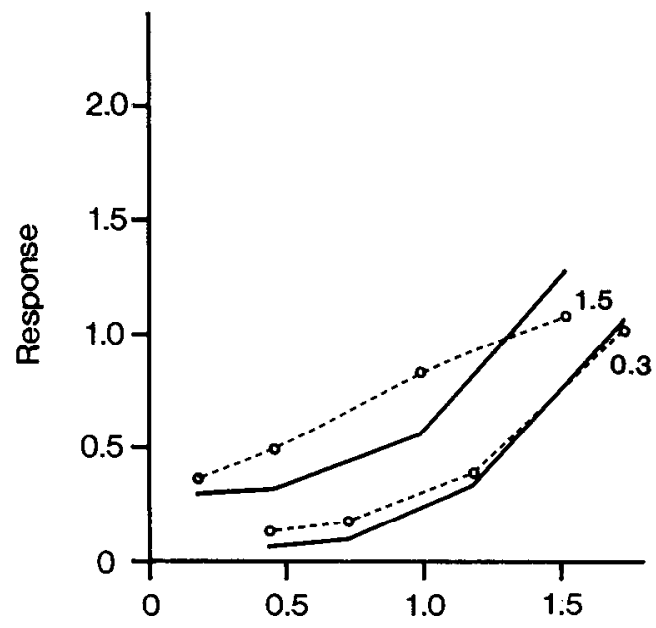

B. Rapidly adapting afferents

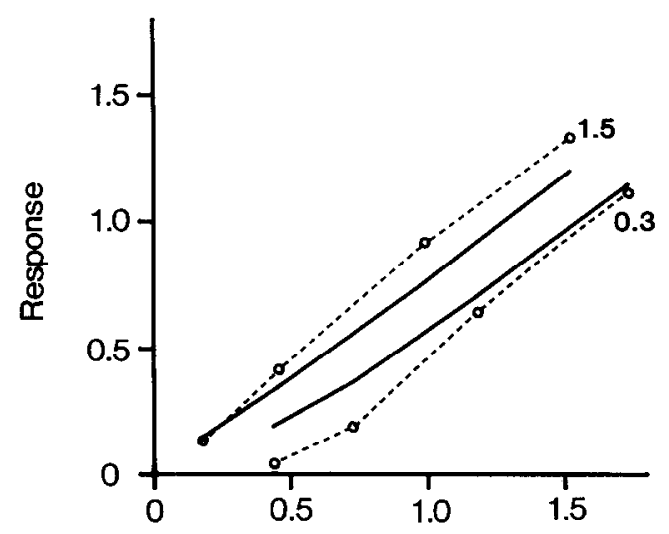

C. Pacinian afferents

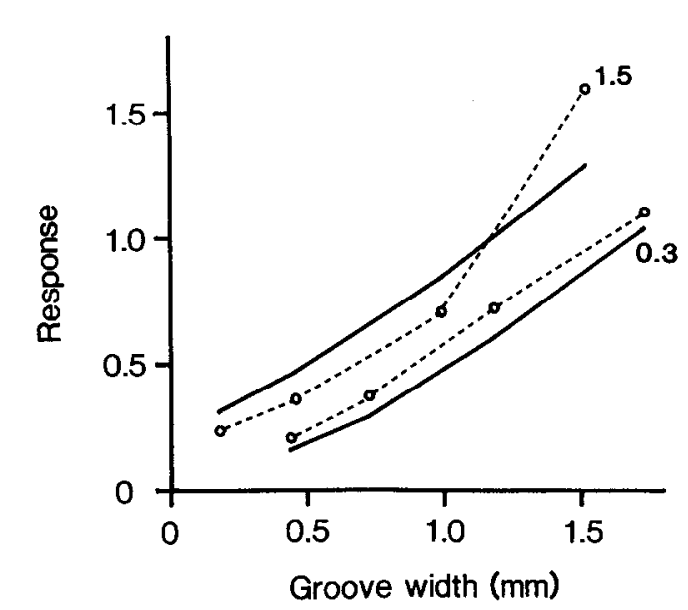

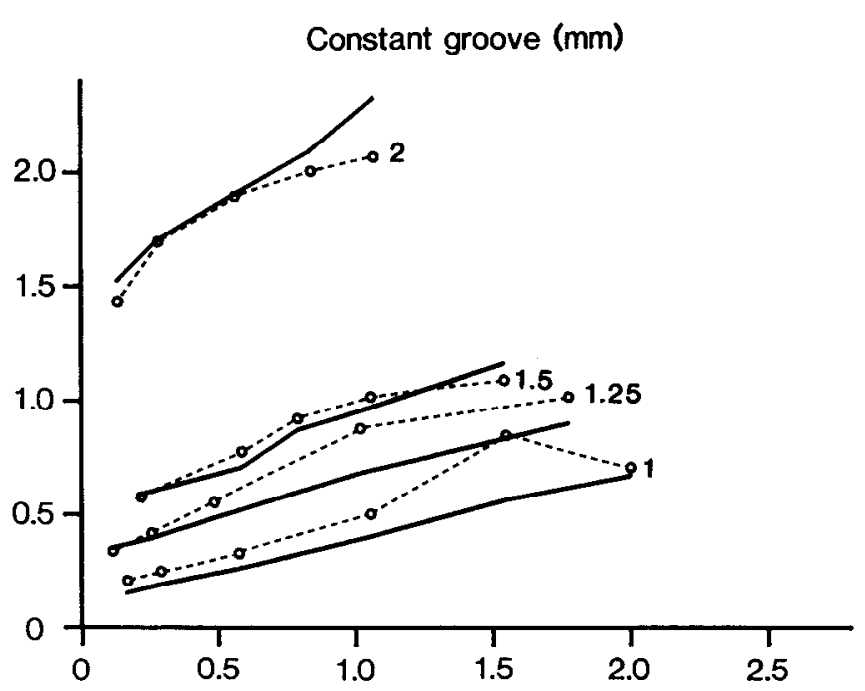
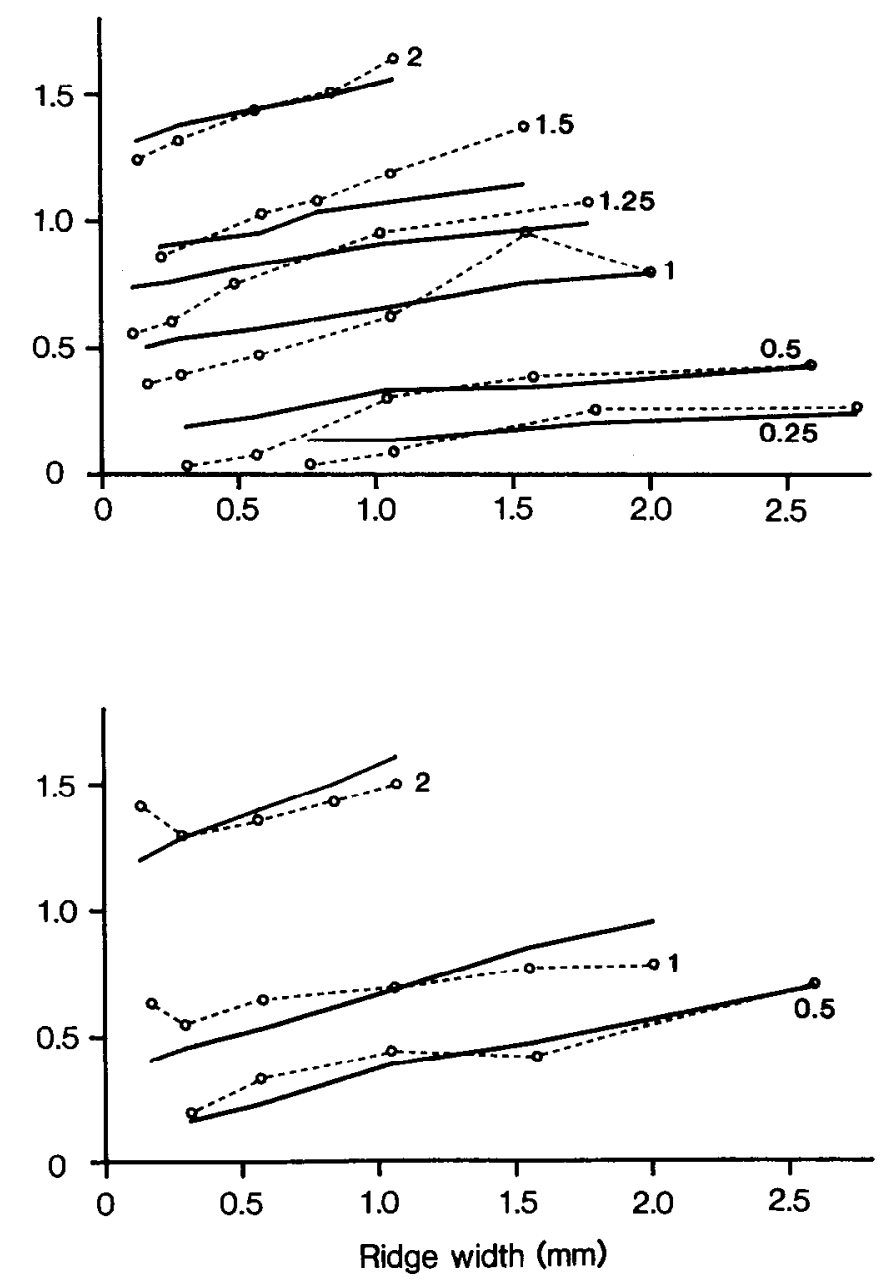

Figure 3. Comparison of responses predicted by the model with experimental values when peak speed remained constant at $80 \mathrm{~mm} / \mathrm{sec}$. The response measure is the mean normalized number of impulses per grating spatial cycle. Experimental values (open circles and broken lines) have been replotted from Figure 1, where the SEs and numbers of afferents are also shown. Solid lines show predictions of the model. 
The values of the constants $a$ in Table 1 show that groove width had a greater effect on SAs than on RAs or PCs. The values of $b$ establish that responses decline with increasing peak temporal frequency of the grating spatial cycles more rapidly for SAs than for PCs, and more rapidly for PCs than for RAs (see Discussion).

\section{Profiles of instantaneous discharge rate}

During the portions of the movement cycle when speed is nearly constant, the equation above gives the incremental number of impulses per grating spatial cycle, $I^{\prime}$ inc, for any values of the stimulus parameters $G, W, S$, and $F$. Here, the instantaneous temporal frequency of the grating spatial cycles $(f)$ has the constant value $F$, so that the incremental instantaneous discharge rate (impulses per second) is simply the product $F I_{\text {inc }}^{\prime}$. During the remainder of the movement cycle, $f$ is changing continuously. Although the equation was derived from data for which $f$ was essentially constant, it is likely to be valid at any instant of time when $f$ is changing (this assumption is justified by the results that follow). That is,

$$
i_{\text {inc }}^{\prime}=c \bar{G}^{a} \exp (-b \vee f) .
$$

On this basis, the incremental instantaneous discharge rate at any point in the movement cycle will be given by the product $f_{i \text { in. }}^{\prime}$. The instantaneous value of $f$ can be calculated readily (see Appendix). The discharge rate discussed above is the incremental component; the background component must be added to obtain the actual discharge rate of the afferent. Details of the computations are given in the Appendix.

The shape of the discharge profile varied with the stimulus parameters and the afferent type. Morley and Goodwin (1987) have illustrated the range of shapes seen by them, which is consistent with the range of shapes seen in our data. The major factors influencing shape are readily appreciated from the model. For RAs, the profiles tended to be close to sinusoidal. For parameters that resulted in higher values of $F$ (high speeds and small spatial periods), the peak flattened slightly because of the factor $\exp (-b \vee F)$. For SAs, the flattening was much more pronounced because of the larger value of $b$, to the extent that, at higher values of $F$, the response exhibited a trough in the region of highest speed. Moreover, the background discharge was significant compared with the incremental discharge, so that the profiles for SAs tended to be much flatter than for RAs. The situation for our PCs was similar to that for the SAs, but the background discharge was smaller, as was the value of $b$. Thus, their profiles tended to be flatter than those for RAs, but not as flat as those for SAs. Figure 5 illustrates 3 afferents from our data; an RA with a profile close to sinusoidal, a PC with a flatter profile, and an SA with an even flatter profile exhibiting a trough in the region of maximum speed. Superimposed on the profiles are the reconstructed responses for the same values of stimulus parameters. Thus, the model provides an adequate description of the instantaneous responses of the afferents during the entire sinusoidal cycle.

\section{Reconstruction of mean cyclic discharge rate}

The mean cyclic response is the average value, over 1 cycle of movement, of the instantaneous discharge rate. As shown previously, this measure differs from the number of impulses per grating spatial cycle. Extension of the model to instantaneous responses (above) enables prediction of the mean cyclic responses. In Figure 6 , the experimental mean cyclic responses are plotted (open circles and broken lines) for both the constant ridge width series and the constant groove width series. These are the same curves as shown in Figure 3 of the previous paper (Sathian et al., 1989) where the SEs and numbers of afferents are also shown. The predictions of the model are easily calculated (see Appendix for details) and are shown by the solid lines. Here, too, the major trends of the data are predicted by the model. This provides additional evidence of the validity of extending the model to instantaneous responses over the entire sinusoidal cycle. The model is particularly valuable in elucidating why the 2 response measures differ. As an illustration, compare Figure $3 C$ (right), where the number of impulses per grating spatial cycle increases as a function of ridge width, and Figure $6 C$ (right), where the mean cyclic response decreases as a function of ridge width. The number of impulses per grating spatial cycle (Fig. 3) is determined by the factor exp $(-b \vee F)$; increasing ridge width decreases $F$ and thus increases the factor. The mean cyclic response (Fig. 6) is determined by the factor $F \exp (-b \vee F)$. With incrcasing ridge width, the increase in $\exp (-b \vee F)$ is less than the corresponding decrease in $F$ so that the product $F \exp (-b \vee F)$ decreases.

Groodwin and Morley (1987a) described the mean cyclic responses of the 3 afferent types to similar stimuli. However, there were 2 major differences in their experiments. First, the spatial features of the stimulus were altered by changing the spatial period of the grating, maintaining the ratio of groove width to ridge width constant at 7 -thus, groove width and ridge width always covaried. Second, a much wider range of peak speeds and peak temporal frequencies were used than in the present study. Thus, a powerful test of the model, derived from the present data, is its ability to reproduce the trends reported by Goodwin and Morley. They show 6 sets of parametric curves for each fiber type, and our equation predicts all the trends for the SAs and RAs, and most of the trends for the PCs (see Discussion). We cannot illustrate all 18 conditions, but Figure 7 shows 4 of the more important reconstructions. Mean cyclic response is shown as a function of peak speed (range, 20-480 $\mathrm{mm} / \mathrm{sec}$ ) when the spatial period of the grating remains constant, and as a function of spatial period when the peak speed remains constant; both RAs (left) and SAs (right) are shown. While RA responses increase with increasing speed (top left), SA responses are essentially invariant with speed (top right). Increasing the spatial period of the grating increases the responses of the RAs (bottom left), and increases the responses of the SAs (bottom right) even more rapidly. These reconstructions are entirely consistent with the data of Goodwin and Morley (compare their figures 5 and 7).

\section{Discussion}

\section{Spatial and temporal factors determining responses}

When a grating moves sinusoidally across the skin, 2 spatial variables (groove width and ridge width) and 2 temporal variables (peak speed and peak temporal frequency of the grating spatial cycles) are pertinent. However, these variables are not independent, and, in the past, it has been difficult to ascertain whether a change in response was due to a change in a spatial variable or to covariation of a temporal variable and vice versa. Our experiments show that the responses of all 3 primary afferent types can be expressed in terms of only 1 spatial variable, namely, groove width $(G)$, and only 1 temporal variable, namely, peak temporal frequency of the grating spatial cycles $(F)$. Ridge width $(W)$ and peak speed $(S)$ only affect the response if 


\section{A. Slowly adapting afferents}

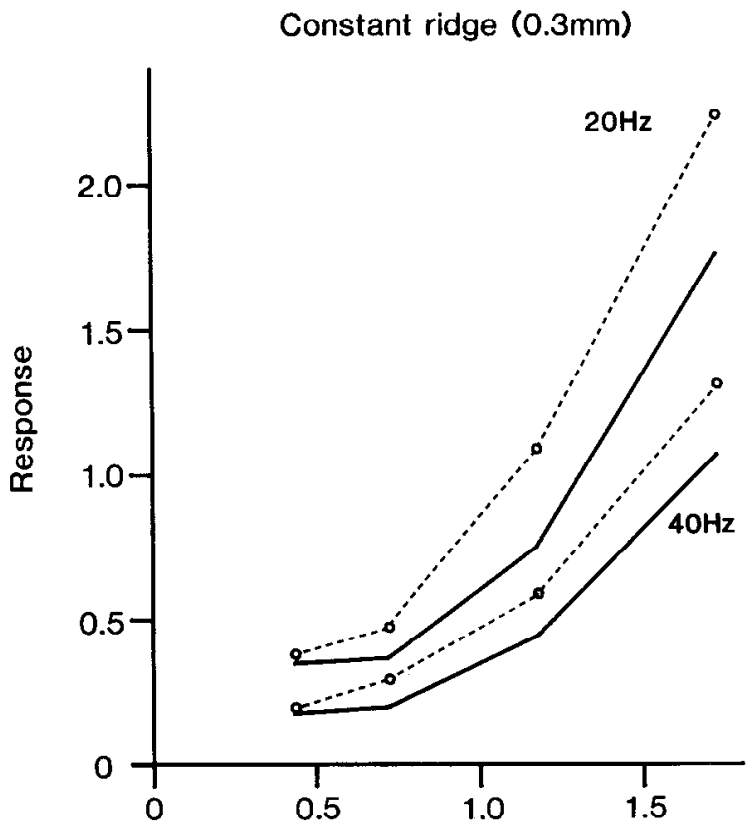

B. Rapidly adapting afferents

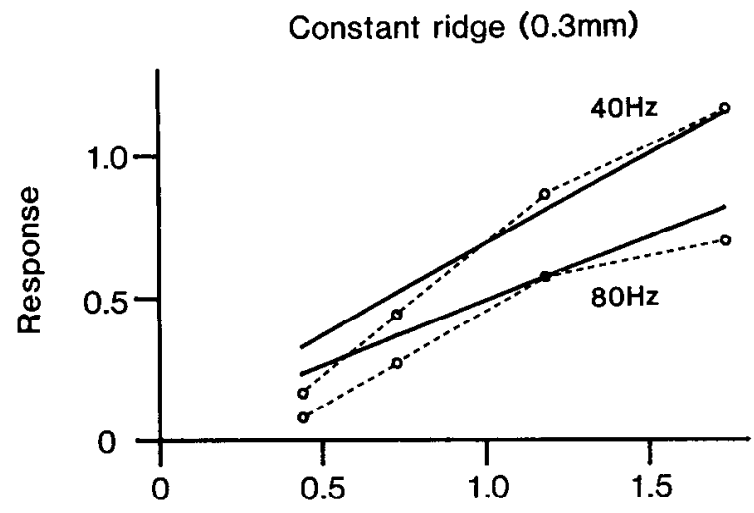

\section{Pacinian afferents}

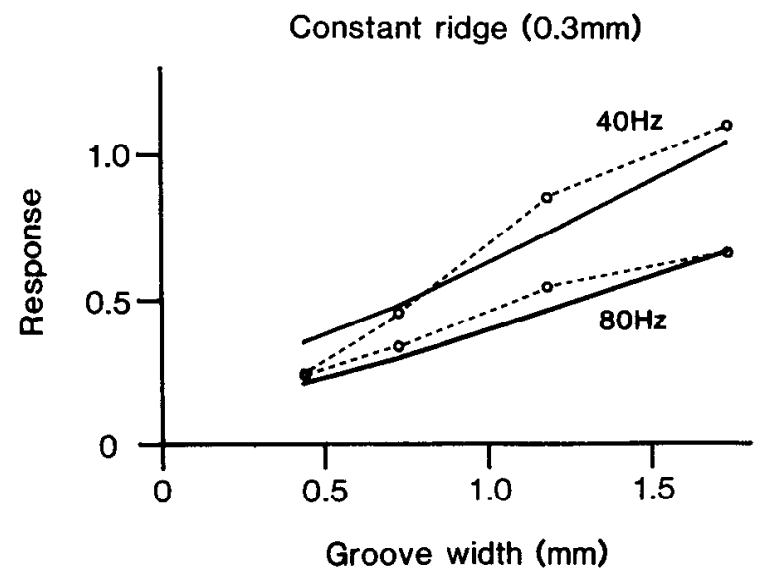

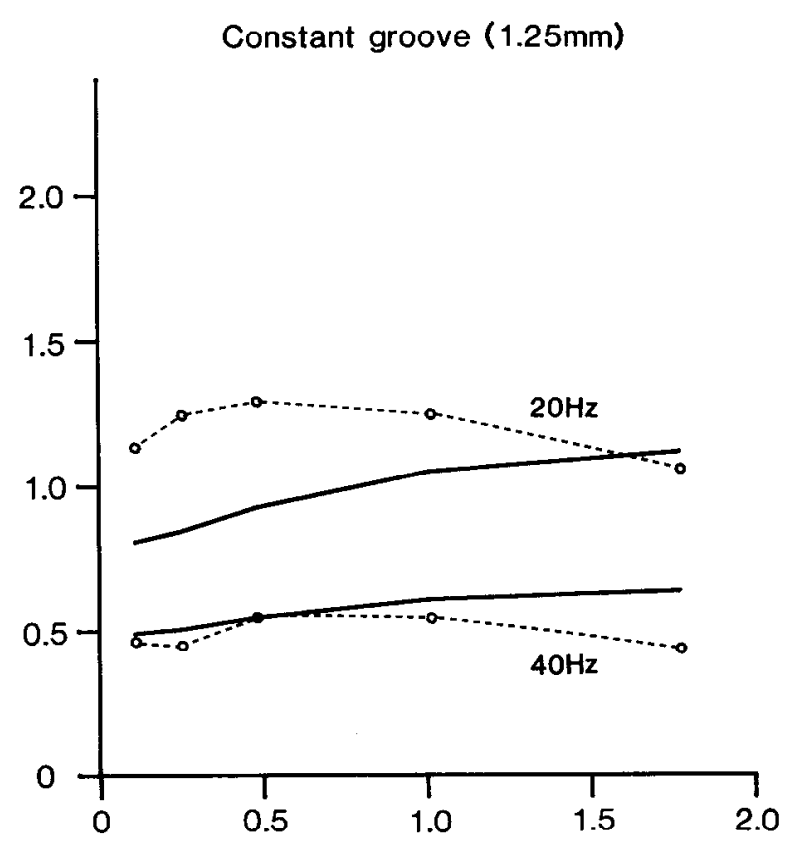
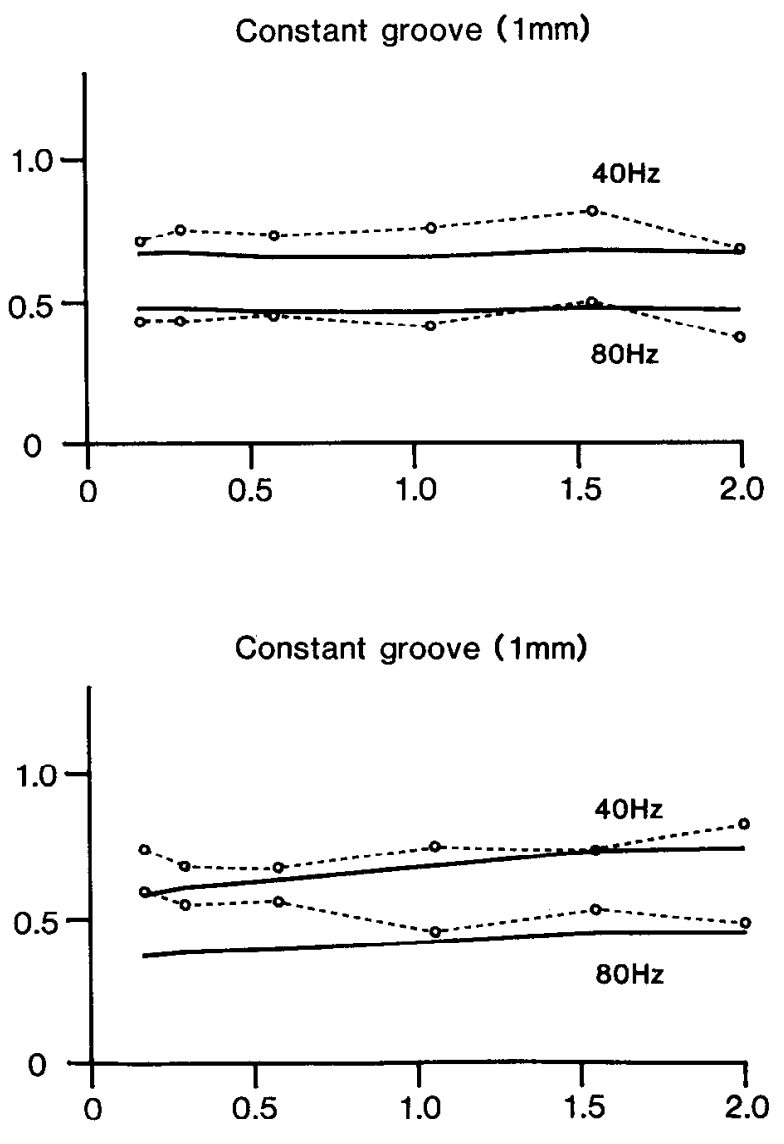

Ridge width (mm)

Figure 4. Comparison of responses predicted by the model with experimental values when the peak temporal frequency of the grating spatial cycles remained constant at the values indicated for each curve. The response measure is the mean normalized number of impulses per grating spatial cycle. Experimental values (open circles and broken lines) have been replotted from Figure 2, where the SEs and numbers of afferents are also shown. Solid lines show predictions of the model. 
RA

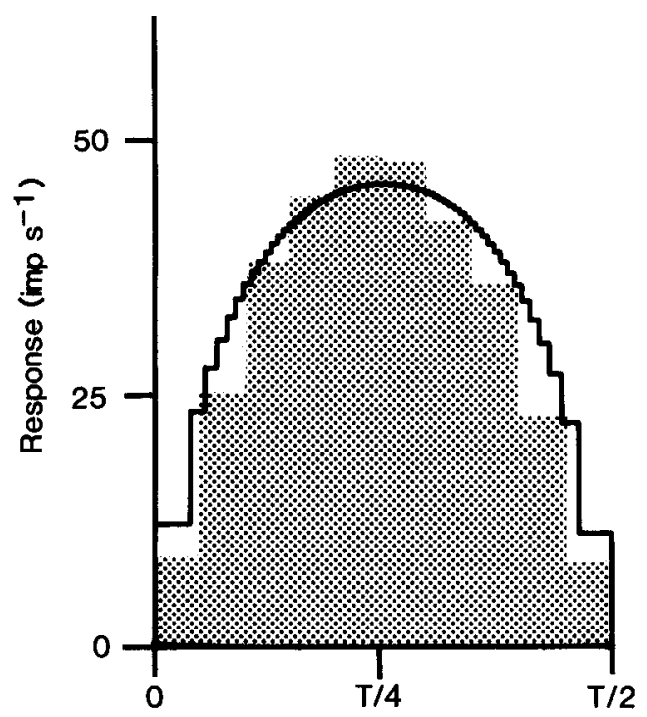

PC

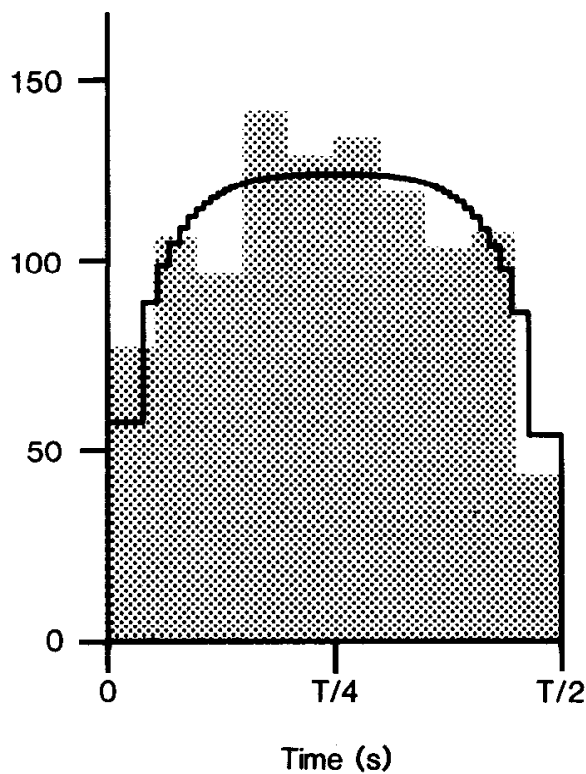

SA

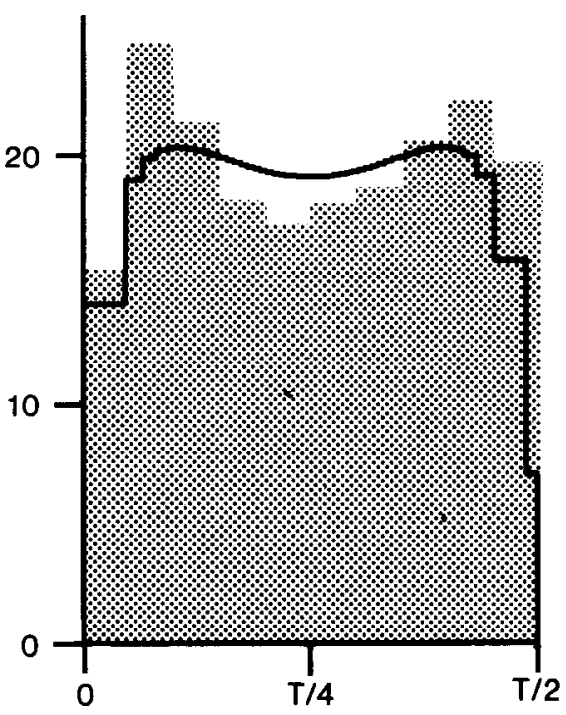

Figure 5. Profiles showing instantaneous responses, during a half-cycle of sinusoidal movement, for 3 afferents. Shading indicates poststimulus time histograms of experimental data, and solid lines show the predictions of the model for the same values of stimulus parameters. Stimulus parameters for the RA are groove $=0.968 \mathrm{~mm}$, ridge $=0.286 \mathrm{~mm}$, peak speed $=50.2 \mathrm{~mm} / \mathrm{sec} ;$ for the PC, groove $=0.968 \mathrm{~mm}$, ridge $=0.286$ $\mathrm{mm}$, peak speed $=160 \mathrm{~mm} / \mathrm{sec}$; and for the $S A$ groove $=1.45 \mathrm{~mm}$, ridge $=0.214 \mathrm{~mm}$, peak speed $=160 \mathrm{~mm} / \mathrm{sec}$. Bin widths and number of trials averaged are $0.250 \mathrm{sec}, n=14(\mathrm{RA}) ; 0.0785 \mathrm{sec}, n=15(\mathrm{PC})$; and $0.0787 \mathrm{sec}, n=18(\mathrm{SA})$. For the predicted responses an arbitrary scaling factor has been applied to match the sensitivity of the particular fiber.

they result in a change in $G$ or $F$. Thus, for example, a change in ridge width has no effect if $G$ and $F$ are held constant.

For all 3 afferent types, response increascd with incrcasing groove width according to a power function. For RAs and PCs, the function was close to linear. For SAs, the groove effect was more pronounced (exponent about 2.6). Why should response be a function of groove width and not ridge width? An intuitive explanation is provided by considering the skin profile under a grating. The skin conforms to the ridges, and is flat in these regions, but "protrudes" into the grooves; the extent of protrusion increases as the groove widens (in the present study the grooves were sufficiently deep that the skin never touched the bottoms). Thus, an increase in groove width increases the curvature and stresses of skin in this region, whereas an increase in ridge width does not affect these parameters. There is evidence in the literature suggesting that mechanoreceptors are excited by local stresses and strains that result from skin curvature (Vierck, 1979; Phillips and Johnson, 198 1a, b; Johansson et al., 1982b; Srinivasan and LaMotte, 1987). Unfortunately, at this stage it is not possible to give a more rigorous explanation as a sufficiently general model of skin mechanics, and the mechanics and transduction processes of the 3 receptor types, does not exist.

For all 3 afferent types, responses decreased as the peak temporal frequency of the grating spatial cycles $(F)$ increased, according to the function $\exp (-b \vee F)$. The decay is most pronounced for the SAs (highest value of $b$ ) and is similar for RAs and PCs ( $b$ is slightly greater for PCs than for RAs). This result is consistent with the data of Goodwin and Morley (1987a). Previous accounts of peripheral neural responses to surfaces moving over the skin have not analyzed the temporal factors in detail (e.g., Lamb, 1983; LaMotte and Srinivasan, 1987a, b). Thus, it is not possible to compare these studies with our data. As each groove of the grating moves onto the contact area, the skin protrudes into the groove with a time course that depends on the skin mechanics. Intuitively, it is clear that as the frequency of occurrence of successive ridges $(F)$ increases, the skin will have less time to protrude into the groove and will thus attain a smaller amplitude of protrusion. This is one factor that results in responses decreasing as $F$ increases; the exact nature of the function will depend on the details of the skin mechanics. A second factor is the temporal filter characteristics of the skin-receptor complex. The filter characteristics of the 3 afferent types have been extensively studied using vibratory stimuli, and these results are considered below.

The equations derived in this study allow us to predict responses when the spatial period of the grating is changed, maintaining the groove width to ridge width ratio constant. These predictions match the experimental data of Goodwin and Morley (1987a) for the SAs and RAs. There is a discrepancy for the PCs in that an increase in groove width increased the responses of our PCs, whereas an increase in spatial period had a variable effect on the responses of PCs studied by Goodwin and Morley, with no overall change in the population response. Our PCs were selected with more rigid criteria for location of the receptive field, resulting in a subpopulation with more consistent responses similar to those of the RAs-see Discussion of previous paper (Sathian et al., 1989). The equations arc consistent with the temporal properties of all 3 afferent types as reported by Goodwin and Morley.

\section{Comparison with responses to vibrating probes}

In previous investigations, there has been difficulty in reconciling the responses to gratings with the responses to vibrating 


\section{A. Slowly adapting afferents}

Constant ridge $(\mathrm{mm})$

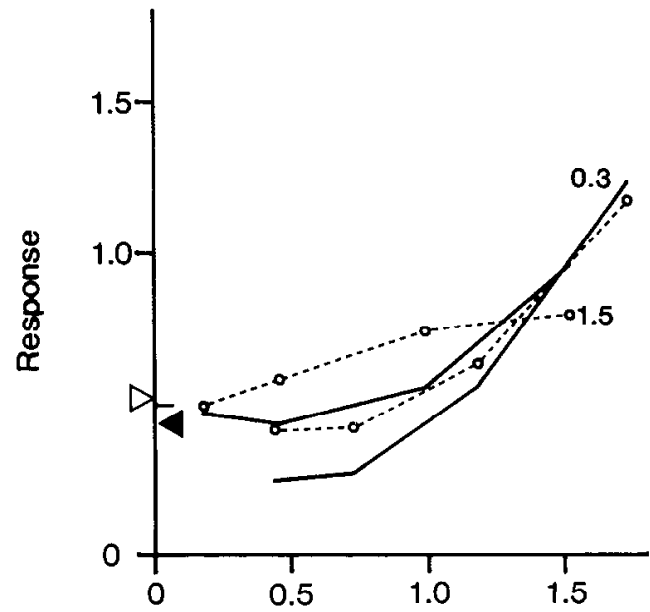

\section{B. Rapidly adapting afferents}

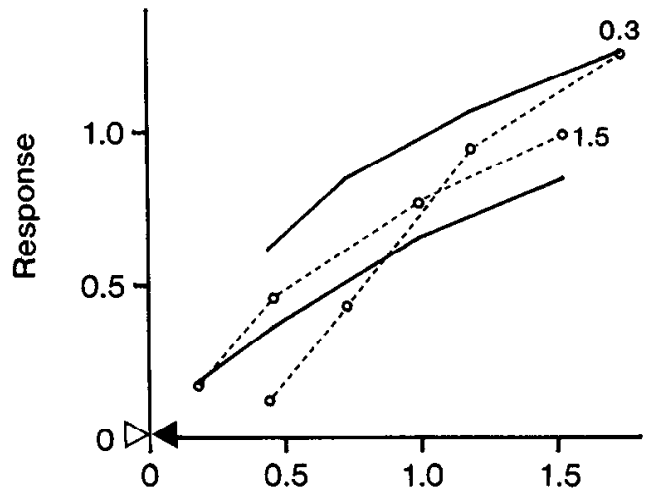

\section{Pacinian afferents}

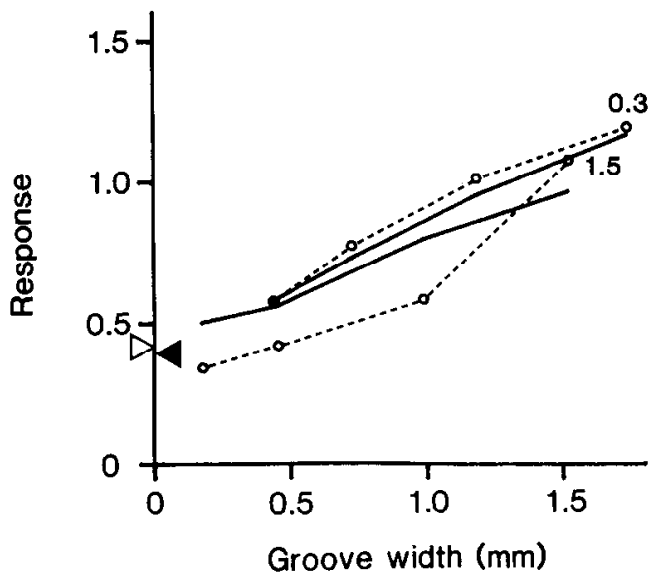

\section{Constant groove $(\mathrm{mm})$}

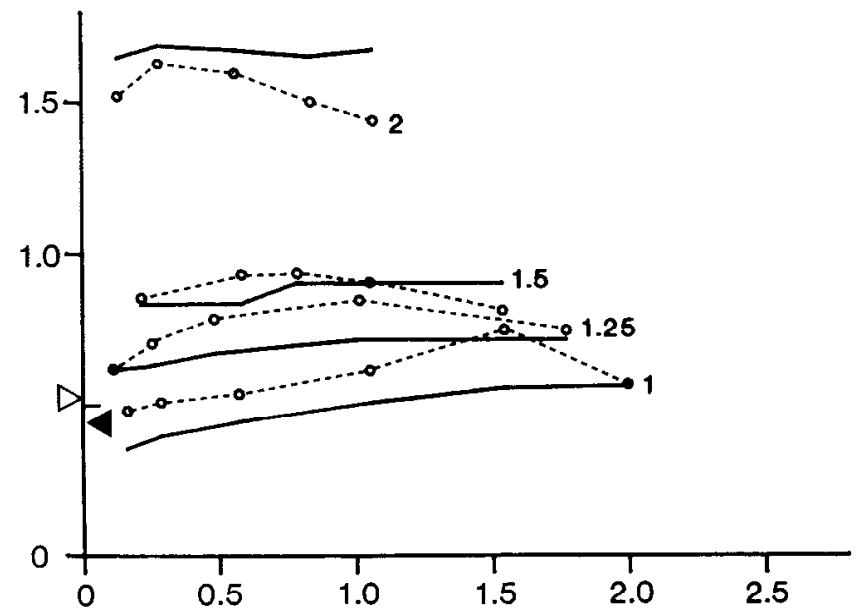




\section{A. Constant spatial period $(\mathrm{mm})$}
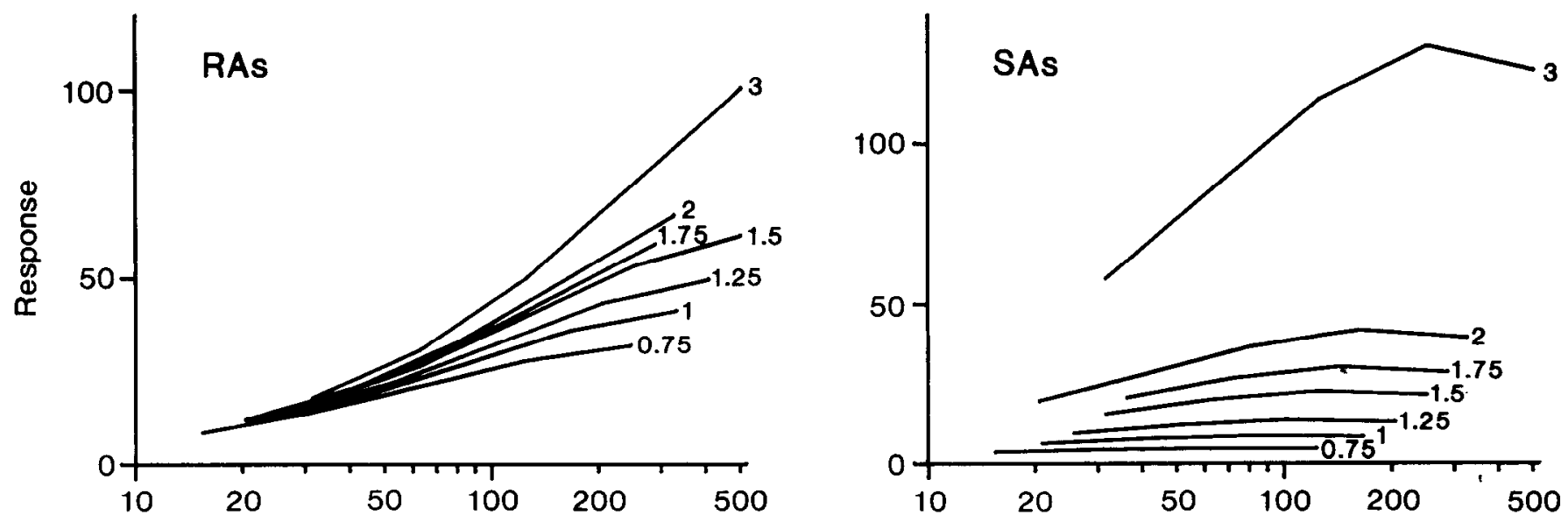

\section{B. Constant peak speed $\left(\mathrm{mm} \mathrm{s}^{-1}\right)$}

Peak speed $\left(\mathrm{mm} \mathrm{s}^{-1}\right)$
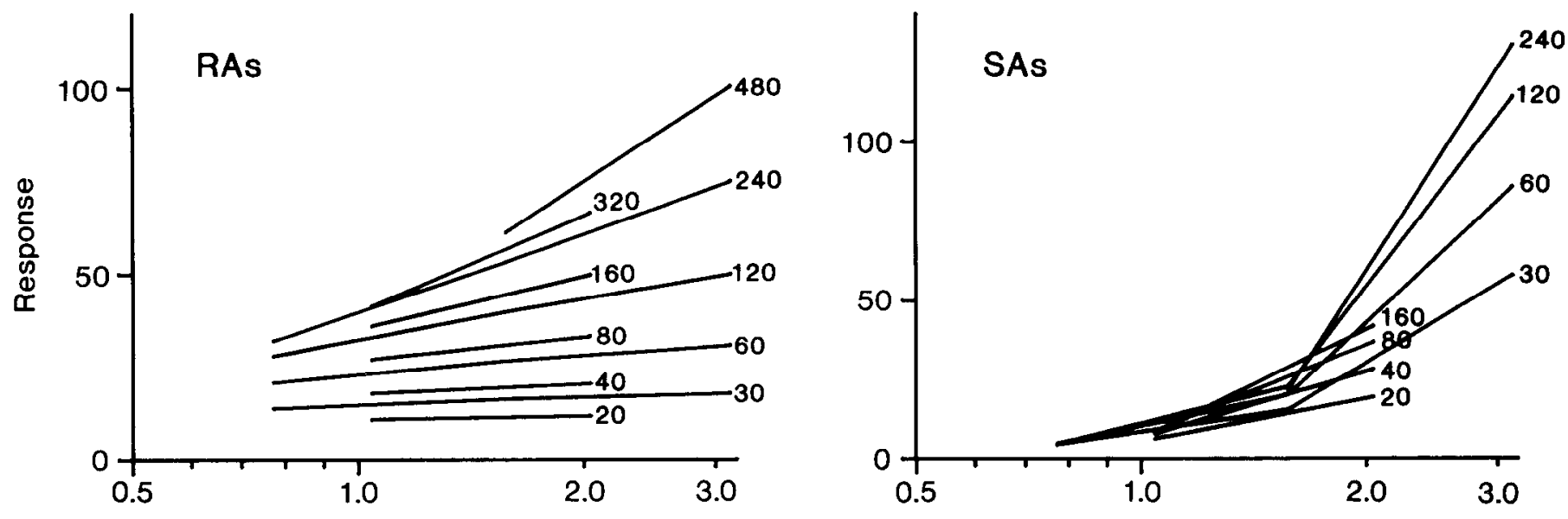

Spatial period $(\mathrm{mm})$

Figure 7. Mean cyclic responses of RAs (left) and SAs (right) predicted by the model. $A$, Spatial period remains constant at the value (mm) indicated next to each curve, and peak speed varies over a range from 20 to $480 \mathrm{~mm} / \mathrm{sec} . B$, Peak speed remains constant, and spatial period varies (both groove width and ridge width vary, their ratio remaining constant at 7 ). For these reconstructions, the actual values of groove widths, ridge widths, and peak speeds used were identical to those used in the experiments of Goodwin and Morley (1987a). The scaling factor for the response axes is arbitrary, and will differ for fibers with different sensitivities.

probes, and apparent conflicts remain. For example, RAs have minimum vibratory thresholds around $30-40 \mathrm{~Hz}$ (Talbot et al., 1968), but their responses to gratings do not reflect such tuning and, in fact, increase as the peak temporal frequency of the grating spatial cycles increases up to at least $320 \mathrm{~Hz}$ (Goodwin and Morley, 1987a). A quantitative comparison of responses to moving gratings with those to vibrating probes cannot be made without a detailed spatiotemporal model of skin and receptor mechanics. However, the temporal factors in both cases must derive from the same fundamental mechanism. We have shown that, for gratings, the temporal factor is adequately described by a function of the form $\exp (-b \vee F)$. How can this be reconciled with the classical vibratory tuning curves?

In Figure 8, vibratory responses measured in the human by Johansson et al. (1982a) have been replotted. The broken lines in the top left figure show responses of RAs at 3 amplitudes.
The response measure is the number of impulses per vibratory cycle, which is analogous to the number of impulses per grating spatial cycle used by us. The solid line is the function $k \exp (-b \vee F)$, with $b=0.130$ (the value for our RAs), and $k$ arbitrarily chosen to superimpose on the broken line. At the low amplitude, the response is entirely consistent with the classical tuning curve. However, at the high amplitude the curve is dramatically shifted so that for frequencies above $2 \mathrm{~Hz}$, response decreases monotonically with frequency (frequencies below this are not significant in the grating experiments). The gratings used by us are suprathreshold stimuli, evoking responses ranging up to as many as 9 impulses per grating spatial cycle, and should be compared with the high-amplitude curve. As seen in Figure 8 , despite the different nature of the experiments and the use of humans rather than monkeys, the trends of the curve are satisfactorily described by the exponential function. 
A. RAs

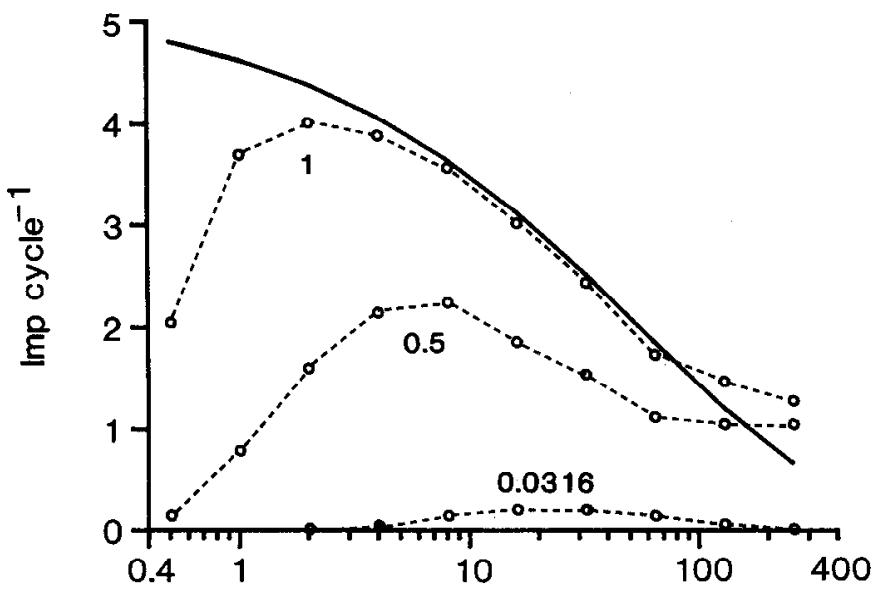

C. PCs

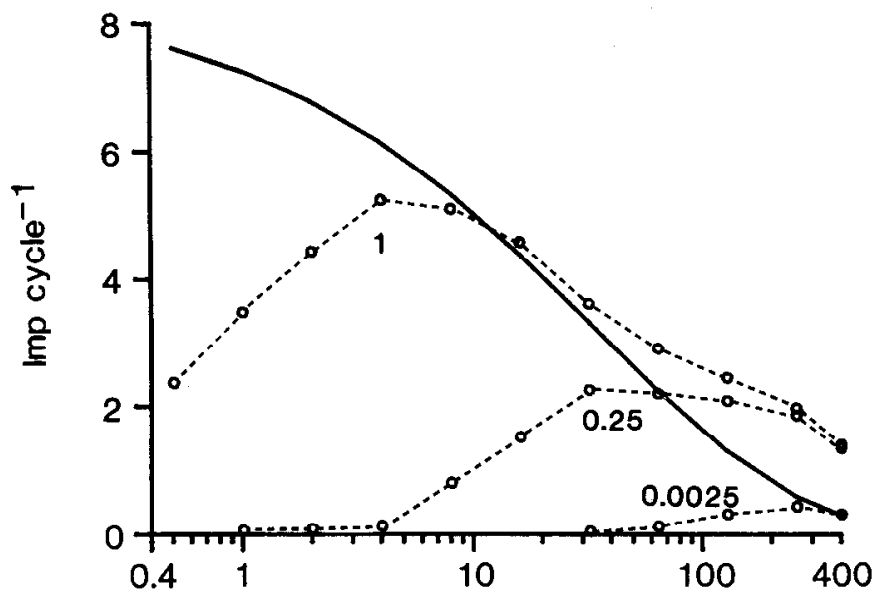

B. RAs

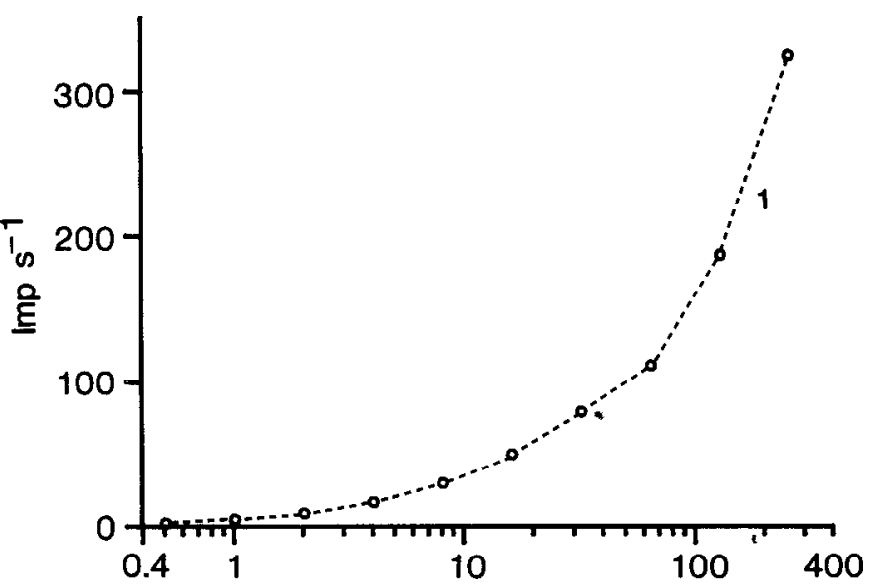

Vibratory frequency $(\mathrm{Hz})$

Figure 8. Responses of the 3 classes of afferents to a probe vibrating sinusoidally. In $A, C$, and $D$, response is measured in impulses per cycle. Data points and broken lines show responses at 3 values of peak-to-peak amplitude (indicated in mm): These have been replotted from the data of Johanson et al. (1982a). Solid lines show functions of the form $k \exp (-b \vee F)$, with the value of $b$ for each afferent type derived from our data (Table 1 ), and the value of the scaling constant $k$ chosen to match the vibratory data. In $B$, the response measure is the mean discharge rate, in impulses per second, obtained from $A$ by the product of the vibratory frequency and the number of impulses per cycle (amplitude is $1 \mathrm{~mm}$ ).

Another difficulty in interpreting tuning curves has been discussed previously by Goodwin and Pierce (1981), who emphasized that a peak entrainment threshold at $30-40 \mathrm{~Hz}$ cannot be looscly cxtrapolated to a maximum response at $30-40 \mathrm{~Hz}$, regardless of the response measure. In Figure $8 B$, the response at an amplitude of $1 \mathrm{~mm}$ has been plotted with the response measure as the mean number of impulses per second, which is analogous to our mean cyclic response. Again, this is consistent with our data; even for vibratory stimuli, the mean response of RAs increases monotonically with frequency.

For PCs (Fig. 8C), the situation is similar, but here there is an even greater shift from the curve for an amplitude of 0.0025 $\mathrm{mm}$ (which has a classical $256 \mathrm{~Hz}$ tuning point) to the curve for an amplitude of $1 \mathrm{~mm}$. For high amplitudes of vibration, the frequency characteristics of the RAs (Fig. 8A) and the PCs (Fig. $8 C$ ) are similar, as is the case in our data. As the amplitude of vibration increases, the curve for the $\mathrm{PC}$ responses tends towards a curve that matches the exponential decay derived from the grating experiments. The SA characteristics (Fig. 8D) also change with amplitude and at higher amplitudes are consistent with the more rapidly decreasing exponential derived from our data.

We do not wish to imply that our model can be generalized to vibratory tuning curves but merely wish to demonstrate that the exponential decay is not in conflict with suprathreshold vibratory responses.

\section{Responses to a smooth surface}

A smooth surface evoked virtually no response in RAs but did evoke a significant response in SAs and PCs. This is not surprising since, even though a smooth surface is devoid of grooves, the coefficient of friction between the surface and the skin is not zero. Thus, tangential motion over the skin ridges will produce stresses that may result in a response. Presumably the static 
components of these frictional stresses produced the large SA responses, and low-amplitude, high-frequency dynamic components produced the large $\mathrm{PC}$ responses. We cannot exclude the possibility that artifacts may have made minor contributions to responses. These include factors such as slight variations in contact force or movement speed (which are present during normal human scanning) and vibration in the stimulator.

\section{Appendix: Mathematical Derivations}

\section{Glossary}

The following list defines the symbols used and their units of measurement.

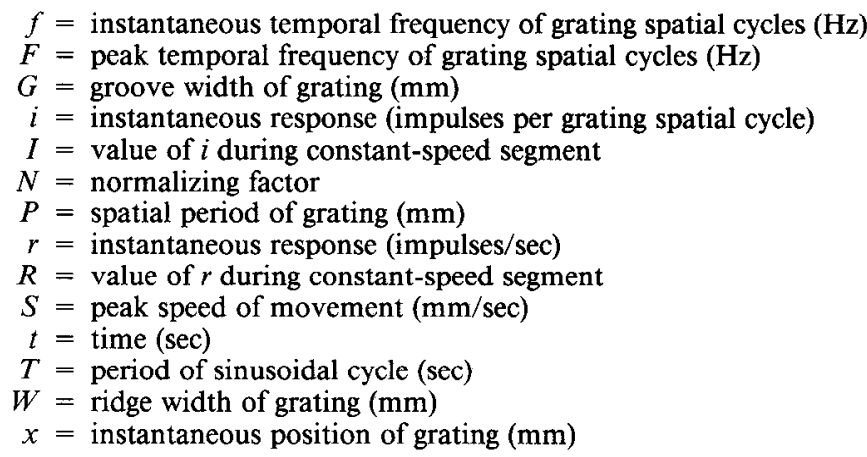

Subscripts bac, inc, and sm denote background, incremental, and smooth, respectively. Superscript prime (') denotes normalization.

\section{Description of stimulus}

Aspects of the stimulus not pursued in detail here are considered in the appendix of Morley and Goodwin (1987). Consider a grating, with spatial period $P$, moving sinusoidally over a receptive field with a peakto-peak amplitude of $80 \mathrm{~mm}$ and a cycle period of $T$. The position and speed at any instant of time, $x$ and $|\dot{x}|$, respectively, may be described by

$$
\begin{gathered}
x=-40 \cos (2 \pi t / T) \\
|\dot{x}|=80 \pi / T|\sin (2 \pi t / T)|
\end{gathered}
$$

Suppose that at time $t$, a point in the receptive field is aligned to some position on the grating, and that at time $t+\delta t$, the point is aligned to the corresponding position in the next spatial cycle of the grating. Then,

$$
P=40|\cos (2 \pi[t+\delta t] / T)-\cos (2 \pi t / T)|
$$

For a given $P$, equation (1) defines $\delta t$ for any $t$, and its reciprocal gives the instantaneous temporal frequency of the grating spatial cycles

$$
f=1 / \delta t
$$

This value of $f$ holds over the entire interval between $t$ and $t+\delta t$, so that $f$ is a steplike function. Note also that the width of the step, $\delta t$, varies during the movement cycle.

When $t=T / 4$ or when $t=3 T / 4$, the speed reaches a maximum given by $S=80 \pi / T$; and from equations (1) and (2), $\int$ reaches a maximum, defined as $F$. Within a region of $42^{\circ}$ about each of these points, the speed remains approximately constant (within $6.6 \%$ of $S$ ) and therefore $f$ remains approximately constant at $F$, given by

$$
S=P F
$$

\section{Regression of data}

Since the fibers' responses to a smooth surface were significant, the response in the region of nearly constant speed is split into 2 components. The first component, which we call the incremental component, is due to the alternating grooves and ridges of the grating. The second component, which we call the background component, reflects a proportion of the response to a smooth surface. As the groove width tends to zero, the incremental component must tend to zero, and the background component must tend to the response to a smooth surface. For a fraction $W / P$ of the grating spatial cycle, the skin is in contact with a ridge, so that the background response may be considered as this fraction of the response to a smooth surface. Thus,

$$
R=R_{\mathrm{inc}}+R_{\mathrm{bac}} \quad \text { and } \quad R_{\mathrm{bac}}=(W / P) R_{\mathrm{sm}}
$$

Therefore,

$$
R=R_{\mathrm{inc}}+(W / P) R_{\mathrm{sm}}
$$

To obtain the number of impulses per grating spatial cycle, the response in impulses per second is simply divided by the temporal frequency of the grating spatial cycles, which in this region is constant at $F$. That is, dividing equation (3) by $F$ gives

$$
I=I_{\text {inc }}+I_{\mathrm{bac}}
$$

where

$$
I_{\text {bac }}=(W / P F) R_{\mathrm{sm}}
$$

Before pooling data, the response of each fiber was normalized by dividing by the average response of that fiber to a preselected block of gratings (see text for details). That is,

$$
N=(1 / n) \Sigma I \quad \text { for } n \text { chosen gratings }
$$

Dividing equation (4) by $N$ gives

$$
I^{\prime}=I_{\text {inc }}^{\prime}+I_{\text {bac }}^{\prime}
$$

Therefore,

$$
I_{\text {inc }}^{\prime}=I^{\prime}-I_{\text {bac }}^{\prime}
$$

where

$$
I_{\text {bac }}^{\prime}=(W / P F N) R_{\text {sm }}
$$

Thus, for each afferent at each combination of stimulus parameters, we calculated $I^{\prime}$, the normalized mean number of impulses per grating spatial cycle (averaged over the 2 regions of nearly constant speed). From equation (6), the incremental component of the response was obtained and used to fit the model

$$
I_{\text {inc }}^{\prime}=c G^{a} \exp (-b \sqrt{ } F)
$$

The constants $c, a$, and $b$ were obtained by nonlinear regression using a direct iterative procedure (Press et al., 1986).

Conversely, the normalized mean number of impulses per grating spatial cycle can be reconstructed from equations (7) and (5) for any of the 3 fiber types for any values of the stimulus parameters.

\section{Reconstruction of response profiles}

The response (impulses per second) at any instant during the sinusoidal cycle can be reconstructed by assuming that equation (7) holds at any instant of time, that is,

$$
i_{\text {inc }}^{\prime}=c G^{a} \exp (-b \vee f)
$$

Also,

$$
i_{\mathrm{bac}}^{\prime}=(W / P f N) r_{\mathrm{sm}} \text { and } i^{\prime}=i_{\text {inc }}^{\prime}+i_{\mathrm{bac}}^{\prime}
$$

The rate in impulses per second is obtained by multiplying $i^{\prime}$ by the instantaneous frequency of the grating spatial cycles, $f$. Thus,

$$
r^{\prime}=f c G^{a} \exp (-b \vee f)+(W / P N) r_{\mathrm{sm}}
$$

Values of $f$ are given by equation (2), and thus $r$ is also a steplike function with variable step width (see Fig. 5). Note that since the background discharge is constant throughout the cycle, $r_{\mathrm{sm}}=R_{\mathrm{sm}}$. The reconstructed response is a normalized response applying to any fiber of that type. A particular fiber's response could be obtained by appropriate scaling depending on the fiber's sensitivity.

\section{Reconstruction of mean cyclic responses}

The mean cyclic response is simply the average response over a cycle of movement and is thus readily obtained from equation (8) by averaging $r^{\prime}$ over a cycle. 


\section{References}

Burr, I. W. (1974) Applied Statistical Methods, Academic, New York. Darian-Smith, 1., and L. E. Oke (1980) Peripheral neural representation of the spatial frequency of a grating moving across the monkey's finger pad. J. Physiol. (Lond.) 309: 117-133.

Freeman, A. W., and K. O. Johnson (1982) Cutaneous mechanoreceptors in macaque monkey: Temporal discharge patterns evoked by vibration, and a receptor model. J. Physiol. (Lond.) 323: $21-41$.

Goodwin, A. W., and J. W. Morley (1987a) Sinusoidal movement of a grating across the monkey's fingerpad: Representation of grating and movement features in afferent fiber responses. J. Neurosci. 7: $2168-2180$.

Goodwin, A. W., and J. W. Morley (1987b) Sinusoidal movement of a grating across the monkey's fingerpad: Effect of contact angle and force of the grating on afferent fiber responses. J. Neurosci. 7:21922202.

Goodwin, A. W., and M. E. Pierce (1981) Population of quickly adapting mechanoreceptive afferents innervating monkey glabrous skin: Representation of two vibrating probes. J. Neurophysiol. 45: 243253.

Goodwin, A. W., B. D. Youl, and N. P. Zimmerman (1981) Single quickly adapting mechanoreceptive afferents innervating monkey glabrous skin: Response to two vibrating probes. J. Neurophysiol. 45: 227-242.

Johansson, R. S. (1978) Tactile sensibility in the human hand: Receptive field characteristics of mechanoreceptive units in the glabrous skin area. J. Physiol. (Lond.) 281: 101-123.

Johansson, R. S., U. Landström, and R. Lundström (1982a) Responses of mechanoreceptive afferent units in the glabrous skin of the human hand to sinusoidal skin displacements. Brain Res. 244: 1725

Johansson, R. S., U. Landström, and R. Lundström (1982b) Sensitivity to edges of mechanoreceptive afferent units innervating the glabrous skin of the human hand. Brain Res. 244: 27-32.

Johnson, K. O. (1974) Reconstruction of population response to a vibratory stimulus in quickly adapting mechanoreceptive afferent fiber population innervating glabrous skin of the monkey. J. Neurophysiol. 35: 48-72.

Knibestöl, M. (1973) Stimulus-response functions of rapidly adapting mechanoreceptors in the human glabrous skin area. J. Physiol. (Lond.) 232: $427-452$.

Lamb, G. D. (1983) Tactile discrimination of textured surfaces: Peripheral neural coding in the monkey. J. Physiol. (Lond.) 338: 567587.
LaMotte, R. H., and M. A. Srinivasan (1987a) Tactile discrimination of shape: Responses of slowly adapting mechanoreceptive afferents to a step stroked across the monkey fingerpad. J. Neurosci. $7: 1655-$ 1671.

LaMotte, R. H., and M. A. Srinivasan (1987b) Tactile discrimination of shape: Responses of rapidly adapting mechanoreceptive afferents to a step stroked across the monkey fingerpad. J. Neurosci. 7: 16721681 .

Morley, J. W., and A. W. Goodwin (1987) Sinusoidal movement of a grating across the monkey's fingerpad: Temporal patterns of afferent fiber responses. J. Neurosci. 7: 2181-2191.

Phillips, J. R., and K. O. Johnson (1981a) Tactile spatial resolution. II. Neural representation of bars, edges, and gratings in monkey primary afferents. J. Neurophysiol. 46: 1192-1203.

Phillips, J. R., and K. O. Johnson (1981b) Tactile spatial resolution. III. A continuum mechanics model of skin "predicting mechanoreceptor responses to bars, edges, and gratings. J. Neurophysiol. 46: $1204-1225$.

Press, W. H., B. P. Flannery, S. A. Teukolsky, and W. T. Vetterling (1986) Numerical Recipes. The Art of Scientific Computing, Cambridge U. P., Cambridge, UK.

Pubols, B. H., Jr., and L. M. Pubols (1976) Coding of mechanical stimulus velocity and indentation depth by squirrel monkey and raccoon glabrous skin mechanoreceptors. J. Neurophysiol. 39: 773-787.

Sathian, K., A. W. Goodwin, K. T. John, and I. Darian-Smith (1989) Perceived roughness of a grating: Correlation with responses of mechanoreceptive afferents innervating the monkey's fingerpad. J. Neurosci. 9: 1273-1279.

Srinivasan, M. A., and R. H. LaMotte (1987) Tactile discrimination of shape: Responses of slowly and rapidly adapting mechanoreceptive afferents to a step indented into the monkey fingerpad. J. Neurosci. 7: 1682-1697.

Talbot, W. H., I. Darian-Smith, H. H. Kornhuber, and V. B. Mountcastle (1968) The sense of flutter-vibration: Comparison of the human capacity with response patterns of mechanoreceptive afferents from the monkey hand. J. Neurophysiol. 31: 301-334.

Vierck, C. J. (1979) Comparison of punctate, edge and surface stimulation of peripheral, slowly-adapting, cutaneous afferent units of cat. Brain Res. 175: 155-159.

Warren, S., H. A. Hamalainen, and E. P. Gardner (1986) Coding of the spatial period of gratings rolled across the receptive fields of somatosensory cortical neurons in awake monkeys. J. Neurophysiol. 56: 623-639. 